\title{
Monocyte-derived alveolar macrophage apolipoprotein E participates in pulmonary fibrosis resolution
}

\author{
Huachun Cui,, Dingyuan Jiang, ${ }^{1,2}$ Sami Banerjee, ${ }^{1}$ Na Xie, ${ }^{1}$ Tejaswini Kulkarni, ${ }^{1}$ Rui-Ming Liu, ${ }^{1}$ \\ Steven R. Duncan, ${ }^{1}$ and Gang Liu' \\ 'Division of Pulmonary, Allergy, and Critical Care Medicine, Department of Medicine, University of Alabama at Birmingham, \\ Birmingham, Alabama, USA. 'Department of Pulmonary and Critical Care Medicine, Center of Respiratory Medicine, China- \\ Japan Friendship Hospital, National Clinical Research Center for Respiratory Diseases, Beijing, China.
}

\begin{abstract}
Recent studies have presented compelling evidence that it is not tissue-resident, but rather monocyte-derived alveolar macrophages (TR-AMs and Mo-AMs, respectively) that are essential to development of experimental lung fibrosis. However, whether apolipoprotein E (ApoE), which is produced abundantly by Mo-AMs in the lung, plays a role in the pathogenesis is unclear. In this study, we found that pulmonary ApoE was almost exclusively produced by Mo-AMs in mice with bleomycin-induced lung fibrosis. We showed that, although ApoE was not necessary for developing maximal fibrosis in bleomycin-injured lung, it was required for the resolution of this pathology. We found that ApoE directly bound to Collagen I and mediated Collagen I phagocytosis in vitro and in vivo, and this process was dependent on low-density lipoprotein receptor-related protein 1 (LPR1). Furthermore, interference of ApoE/LRP1 interaction impaired the resolution of lung fibrosis in bleomycin-treated WT mice. In contrast, supplementation of ApoE promoted this process in $\mathrm{ApoE}^{-/-}$animals. In conclusion, Mo-AM-derived ApoE is beneficial to the resolution of lung fibrosis, supporting the notion that Mo-AMs may have distinct functions in different phases of lung fibrogenesis. The findings also suggest a potentially novel therapeutic target for treating lung fibrosis, to which effective remedies remain scarce.
\end{abstract}

Conflict of interest: The authors have declared that no conflict of interest exist.

Copyright: (c) 2020, American Society for Clinical Investigation.

Submitted: October 25, 2019 Accepted: January 30, 2020 Published: March 12, 2020.

Reference information: /CI Insight. 2020;5(5):e134539.

https://doi.org/10.1172/jici.

insight.134539.

\section{Introduction}

Pulmonary fibrosis is typically characterized by progressive and irreversible lung scarring, which eventually leads to respiratory failure and death (1). Heightened fibrotic responses of interstitial mesenchymal cells to diverse stresses and injuries, as well as impaired alveolar epithelial regeneration are well-established underlying mechanisms $(1,2)$. The pathogenesis has also been ascribed to insufficient or defective resolution of the established fibrotic lesions in the lung (3).

Numerous cell types have been implicated in pulmonary fibrosis. Alveolar macrophages (AMs) have long been known to play a crucial role in the development of lung fibrosis by differentiating into a profibrotic M2 phenotype, a mechanism that was well received in the past (4-7). However, this paradigm is conceptually imprecise because of lacking consideration of the AM heterogeneity present during lung injury and repair (8). With the application of multiparameter flow cytometry, in combination with next-generation RNA sequencing (RNA-seq) and advanced RNA-seq at the single cell level, recent studies have started to unfold the nuances of AMs in this pathology $(6,8-14)$. Evidence shows compellingly that there are 2 ontogenetically distinct subgroups of AMs during fibrogenesis in the lung, namely tissue-resident AMs (TR-AMs) and monocyte-derived AMs (Mo-AMs) (8, 9, 12, 14-16).

TR-AMs and Mo-AMs differ vastly with respect to their phenotype and transcriptome during fibrotic responses to bleomycin lung injury $(8,9,12,14-16)$. However, Mo-AMs become increasingly indistinguishable from TR-AMs as the lung returns to homeostasis in this model (8). A couple of studies that employed genetic or pharmacological depletion of AM subgroups found that TR-AMs seemed to lack a role in bleomycin-induced pulmonary fibrosis. In contrast, ample evidence indicates that Mo-AMs are critical for the maximal development of lung fibrosis in this model $(8,10,16-18)$.

Transcriptomic analysis has attributed the role of Mo-AMs to a handful of putative profibrotic mediators (8). However, other types of cells in the lung, such as myofibroblasts, perhaps express even more 
of these mediators, raising questions about their relative significance in the Mo-AM contribution to lung fibrosis. Additionally, while the profibrotic role of AMs in developing bleomycin-induced lung fibrosis has been firmly established, there is also evidence indicating that AMs are beneficial during the resolution of lung fibrosis in bleomycin-treated mice $(19,20)$. Thus, there remain many key questions concerning the role of Mo-AMs in different phases of lung injury and repair, along with the mediators that are involved in this process.

In this study, we showed that apolipoprotein E (ApoE), a key molecule involved in lipid metabolism (21), was produced primarily by Mo-AMs during bleomycin lung injury. While ApoE did not play a role in developing the maximal fibrosis in bleomycin-treated lung, it was indispensable to the resolution of this pathology. Mechanistically, we found that ApoE was capable of binding to type I Collagen and mediating collagen phagocytosis via the ApoE receptor, low-density lipoprotein receptor-related protein 1 (LRP1) (22). Our data reveal that a previously unappreciated role of the monocyte-derived subset of AMs may not just passively respond with phenotype change during injury, but they also actively participate in resolving fibrosis and maintaining lung homeostasis.

\section{Results}

$A p o E$ is one of the most differentially expressed signature markers in Mo-AMs versus TR-AMs. Recent studies have provided compelling evidence that AMs from mice with bleomycin-induced pulmonary fibrosis are transcriptionally heterogeneous, with the transcriptome of individual AM being largely dictated by its origin. Similar transcriptional heterogeneity has also been found in AMs from idiopathic pulmonary fibrosis (IPF) patients $(14,16)$. These findings collectively indicate that the ontogenetically different AM subtypes may have a distinct role in the pathogenesis of this disease.

Upon survey of the transcriptomes of Mo-AMs and TR-AMs from mice that were treated intratracheally (i.t.) with bleomycin, we were particularly interested in ApoE because (a) it is one of the most differentially expressed genes in Mo-AMs as compared with TR-AMs from bleomycin-treated lungs and (b) its abundance in Mo-AMs is much greater than several previously defined profibrotic mediators in this AM subtype, such as PDGF and Collagen VI (10, 23, 24).

Given this information, we hypothesized that Mo-AM ApoE may have a critical role in pulmonary fibrosis. We first validated the RNA-seq results by real-time PCR, which showed that the ApoE level in Mo-AMs was nearly 120-fold greater than that in TR-AMs (Figure 1A). This difference even dwarfs that of CX3CR1, a characteristic marker of Mo-AMs (13) (Figure 1A). Of note, a similar level of the pan-macrophage marker MerTK in Mo-AMs and TR-AMs and a minimal expression of the TR-AM marker Siglec $\mathrm{F}$ in Mo-AMs confirm the purity of these AMs subgroups (13) (Figure 1A). Because ApoE is a secreted protein, we examined its levels in bronchoalveolar lavage fluid (BALF) from mice with or without bleomycin-induced pulmonary fibrosis and found that ApoE in BALF from bleomycin-treated lungs was plentiful and readily detected (Figure 1, B and C). However, there was almost no detectable ApoE in BALF from the saline-treated control animals (Figure 1, B and C). As expected, fibronectin (Fn) and osteopontin (OPN), 2 important profibrotic mediators $(11,25)$, were also increased in the BALF from fibrotic lungs (Figure 1C). In contrast to AMs, ApoE expression in alveolar epithelial cells (AECs) and fibroblasts (Fbs) from fibrotic lungs demonstrated little change (Figure 1D). Indeed, Mo-AMs produced the vast majority of pulmonary ApoE in fibrotic lungs, indicated by the finding that Mo-AMs expressed approximately 5 times more of this protein than all other non-Mo-AM lung cells combined (Figure 1E).

Similar to the findings demonstrated in mouse lungs, normal healthy human lungs had little ApoE (Figure 1F). However, ApoE expression was markedly increased and enriched in dense fibrotic areas in IPF lungs (Figure 1F). Taken together, these data suggest that ApoE may play an important role in the pathogenesis of lung fibrosis.

ApoE plays no role in the development of pulmonary fibrosis in bleomycin-treated lungs. Given that ApoE is highly and exclusively produced by Mo-AMs and that Mo-AMs have been found crucial in the development of bleomycin-induced lung fibrosis, we explored the role of ApoE in the pathogenesis of this disorder. WT and $\mathrm{ApoE}^{-/-}$mice were treated i.t. with saline or bleomycin (Figure $2 \mathrm{~A}$ ). We first confirmed that there was no ApoE expression in $\mathrm{ApoE}^{-/-}$lungs or AMs (Figure 2B). We assessed lung fibrosis via histological analysis, Masson's trichrome staining of lung collagen deposition, and analysis of pulmonary hydroxyproline levels at 3 weeks after the treatment, a time point at which the pathology is known to peak in this model. We found, much to our surprise, that there was no difference in bleomycin-induced pulmonary fibrosis in WT and 
A

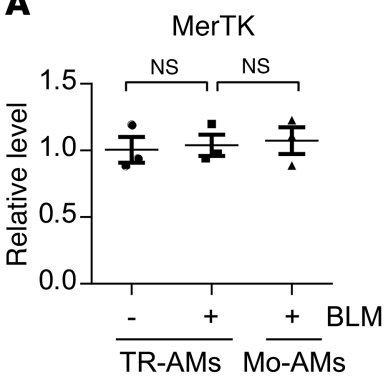

Primary mouse AMs

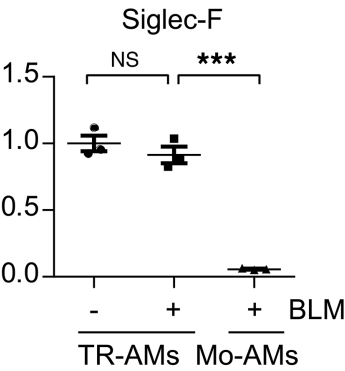

Cx3cr1

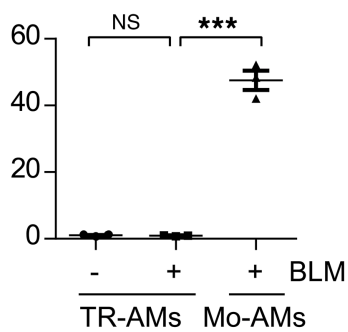

ApoE

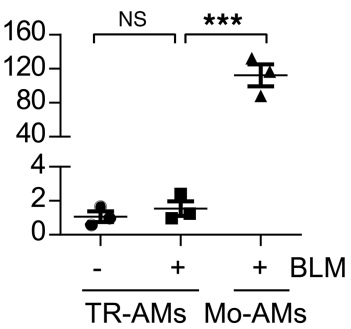

B

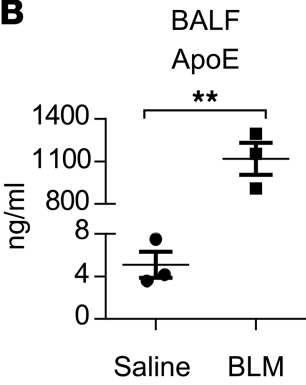

C

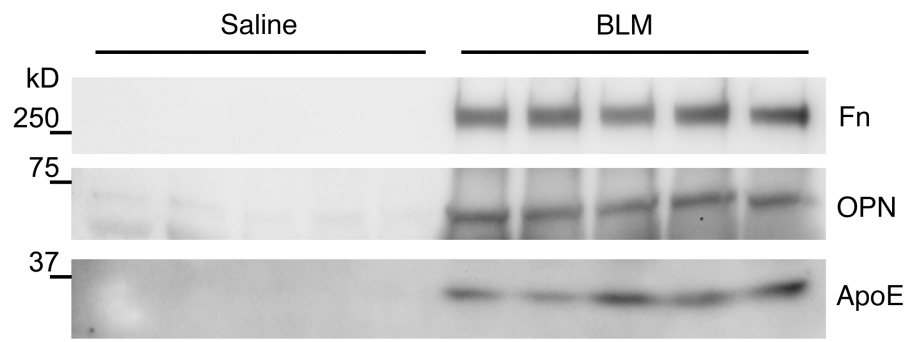

D

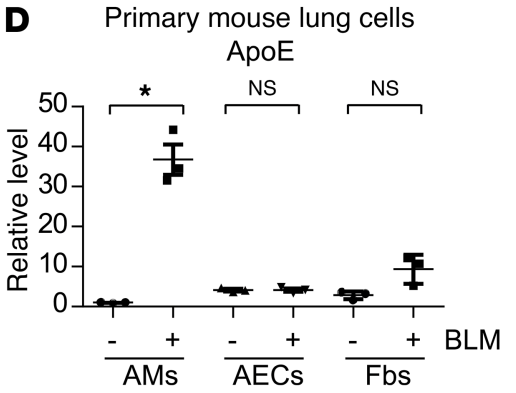

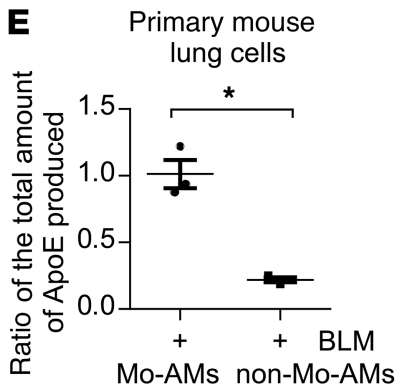

$\mathbf{F}$
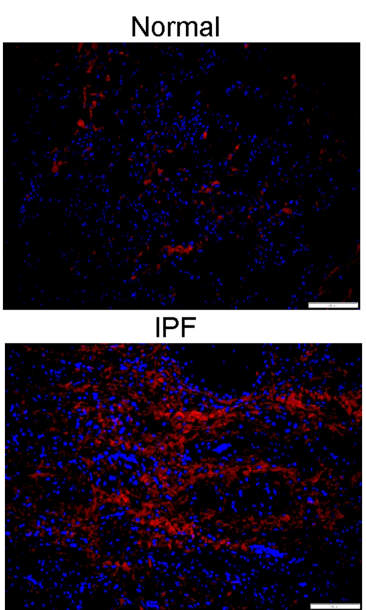

ApoE Nuclei

Figure 1. ApoE is one of the most differentially expressed signature markers in Mo-AMs versus TR-AMs. (A) Eight-week-old C57BL/6 mice were i.t. instilled with saline or bleomycin (BLM, $1.5 \mathrm{U} / \mathrm{kg}$ in $50 \mu \mathrm{L}$ saline). Three weeks after treatment, mice were sacrificed and BALF harvested. BAL cells then underwent fluorescence activated cell sorting for isolation of TR-AMs and Mo-AMs, as described in detail in Methods. Total RNAs of AMs were purified and real-time PCR performed to assess the expression of the indicated genes. $n=3$ mice for each group; mean $\pm S E M ;{ }^{* * *} P<0.001$ by 1 -way ANOVA with Bonferroni's post hoc test. (B) Experiments were done as in A. ApoE levels in BALF were determined by ELISA. $n=3$ mice for each group; mean $\pm \mathrm{SEM}$; ${ }^{*} P<0.01$ by 2 -tailed Student's $t$ test. (C) Experiments were done as in A. BALF protein was precipitated and resolved by SDS-PAGE. Levels of the indicated proteins in BALF were determined by Western blotting. (D) Mice were treated as in A. Lungs were harvested and single cell suspensions prepared. AMs, alveolar epithelial cells (AECs), and fibroblasts (Fbs) were isolated as described in detail in Methods. Total RNAs were purified and real-time PCR performed to assess ApoE expression. $n=3$ mice for each group; mean $\pm S E M$; ${ }^{*} P<0.05$ by 1 -way ANOVA with Bonferroni's post hoc test. (E) Mice were treated as in $\mathbf{A}$. Lungs were harvested and single cell suspension prepared. Mo-AMs were purified by fluorescence activated cell sorting, and all of the remaining lung cells were treated as non-Mo-AMs. Total RNAs from the Mo-AM and non-Mo-AM population were isolated, and the relative level of ApoE in the Mo-AMs and non-Mo-AMs was determined. The ratio of the absolute amount of ApoE produced by the Mo-AMs to that by the non-Mo-AMs was derived by factoring the respective percentage of the Mo-AMs and non-Mo-AMs into this relative level. $n=3$; mean \pm SEM; ${ }^{*} P<0.05$ by 2 -tailed Student's $t$ test. (F) Slices of normal control and IPF lungs were prepared. Immunofluorescence staining and fluorescence microscopy were performed to determine the expression of ApoE. Nuclei were counterstained with DAPI. Original magnification $\times 200$. Scale bars: $100 \mu \mathrm{m}$. 
A
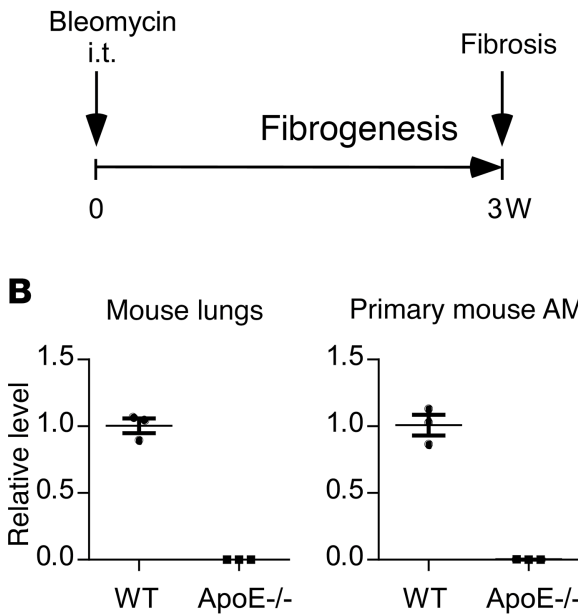

Primary mouse AMs

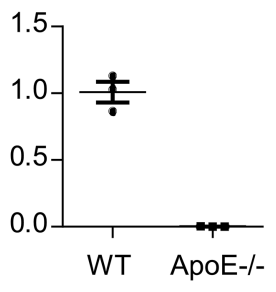

+ BLM

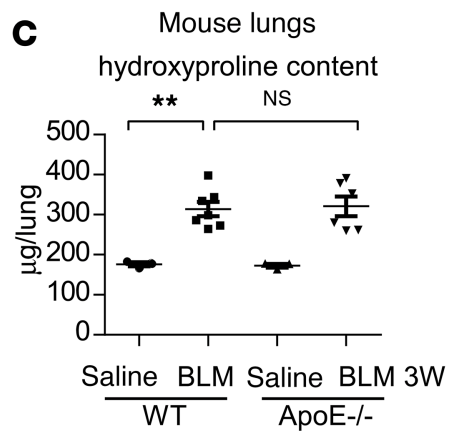

D Saline
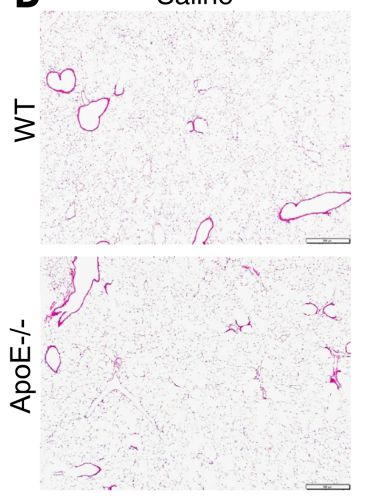

$\mathbf{E}$

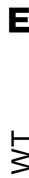

5

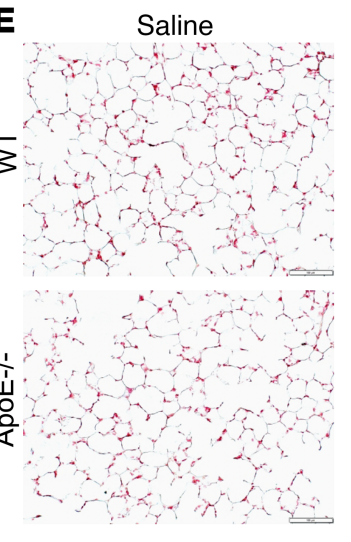

BLM 3W
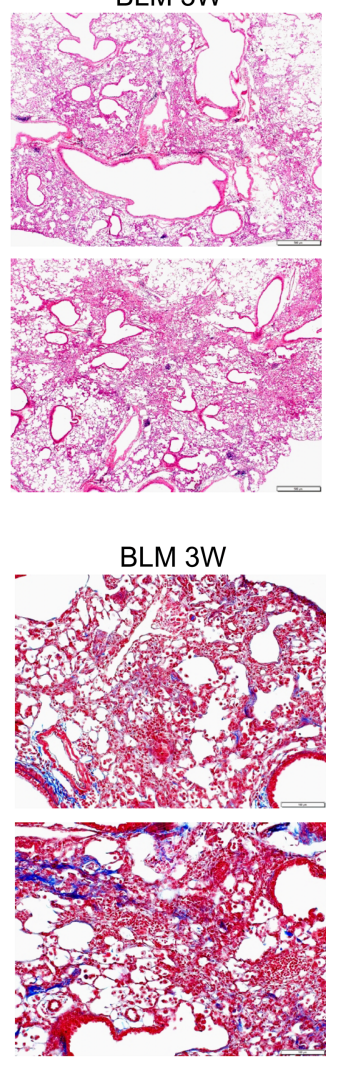

Figure 2. ApoE plays no role in the development of pulmonary fibrosis in bleomycin-treated lungs. (A) Six-week-old WT and $A \mathrm{ApE}^{-/-}$male mice were i.t. instilled with saline or bleomycin (BLM, $1.5 \mathrm{U} / \mathrm{kg}$ in $50 \mu \mathrm{L}$ saline). Three weeks after treatment, mice were sacrificed for AM isolation and fibrosis evaluation. (B) Total RNA of the lungs and AMs from the experiment in $\mathbf{A}$ were purified and real-time PCR performed to assess the expression of ApoE. $n=3$ mice for each group. (C) The levels of hydroxyproline in the lungs were determined. $n=3,7,3,6$ mice for WT Saline, WT BLM, ApoE ${ }^{-1-}$ Saline, and $\mathrm{ApoE}^{-/-} \mathrm{BLM}$, respectively; mean $\pm \mathrm{SEM}$; ${ }^{* *} P<0.01$ by 1-way ANOVA with Bonferroni's post hoc test. (D-E) Representative images of $\mathrm{H} \& \mathrm{E}$ staining (D) and Masson's trichrome staining for collagens (E) of the lungs from this experiment are shown. Original magnification, $\times 40$ (D), and $\times 200$ (E). Scale bars: $500 \mu \mathrm{m}$ (D) and $100 \mu \mathrm{m}(\mathbf{E})$.

$\mathrm{ApoE}^{-/-}$mice (Figure 2, C-E), suggesting that AM ApoE is not required for a fulminant fibrotic response.

$A p o E$ is required for the resolution of bleomycin-induced pulmonary fibrosis. It is well known that bleomycin-induced pulmonary fibrosis resolves spontaneously over time, a process in which deficiency is implicated in the pathogenesis of human $\operatorname{IPF}(3,26)$. Since we failed to find a role of ApoE in the development of fibrosis in bleomycin injured lungs, we next determined if ApoE participated in the resolution of pulmonary fibrosis. WT and $\mathrm{ApoE}^{-/-}$mice were again treated i.t. with saline or bleomycin, but they were assessed at 8 weeks after the treatment, a time when the fibrosis in bleomycin-treated lungs has typically resolved (Figure $3 \mathrm{~A}$ ). At this time point, ApoE levels remained higher in BALF and AMs from bleomycin-injured lungs (Figure 3, B and C). Bleomycin-induced fibrosis mostly resolved in the lungs of WT mice, as evidenced by the close to normal levels of lung hydroxyproline, lung histology, lung collagen deposition, and expression of the profibrotic mediators (Figure 3, D-G). In contrast, there remained a substantial degree of fibrosis in the lungs of bleomycin-treated $\mathrm{ApoE}^{-/-}$animals at this time point (Figure 3, D-G). Actually, the greater fibrotic pathology in ApoE $\mathrm{E}^{-/-}$mice was evident across the entire resolution phase (Supplemental Figure 1, A and B; supplemental material available online with this article; https://doi.org/10.1172/jci.insight.134539DS1). Together, these data suggest that, although ApoE is apparently not involved in the active fibrotic response to bleomycin lung injury, it is required for the resolution of the established fibrotic lesions in the lung.

ApoE directly binds to type I Collagen. The mechanism underlying the resolution of bleomycin-induced lung fibrosis is currently not well understood (3). However, dedifferentiation of myofibroblasts and 
A

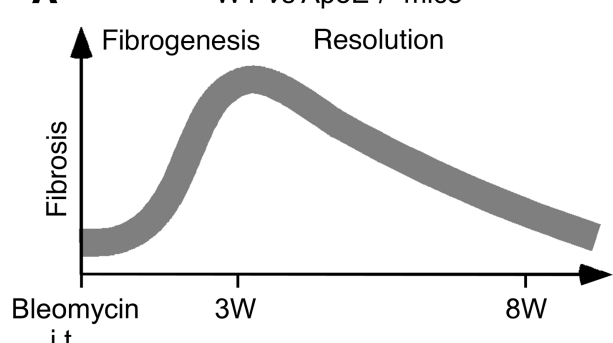

i.t.

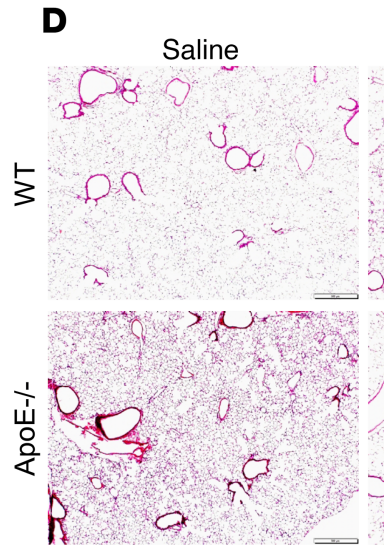

$\mathbf{F}$

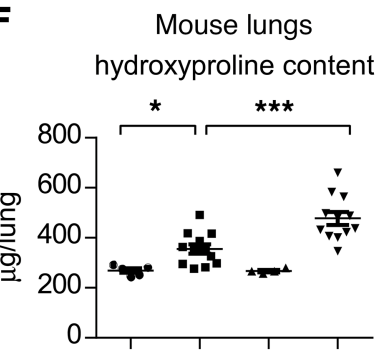

8W Saline BLM Saline BLM
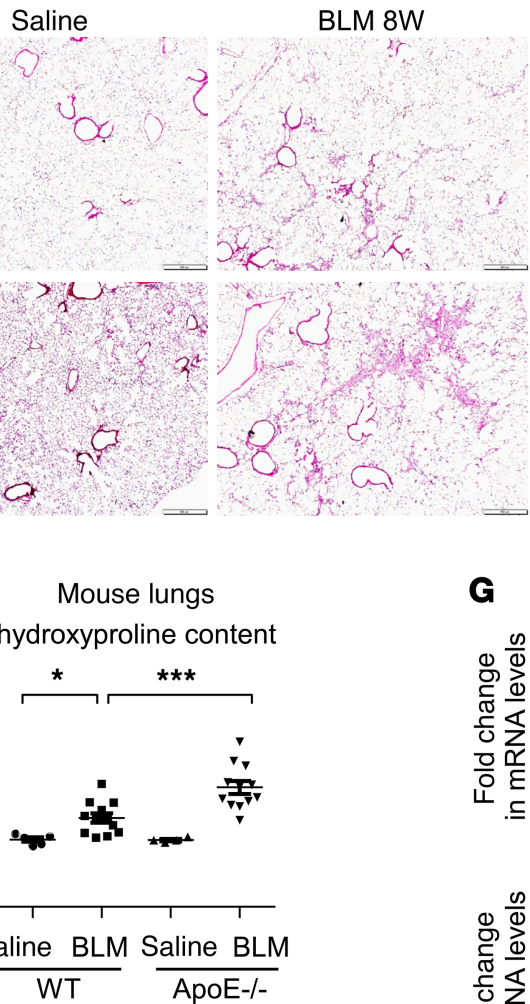

B

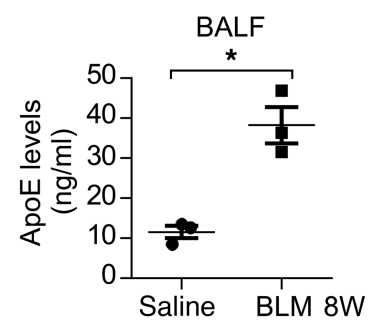

C

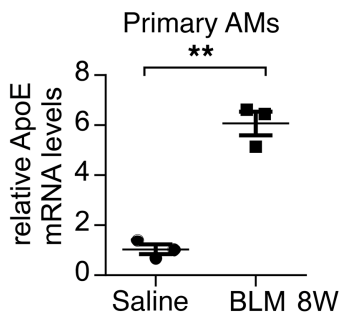

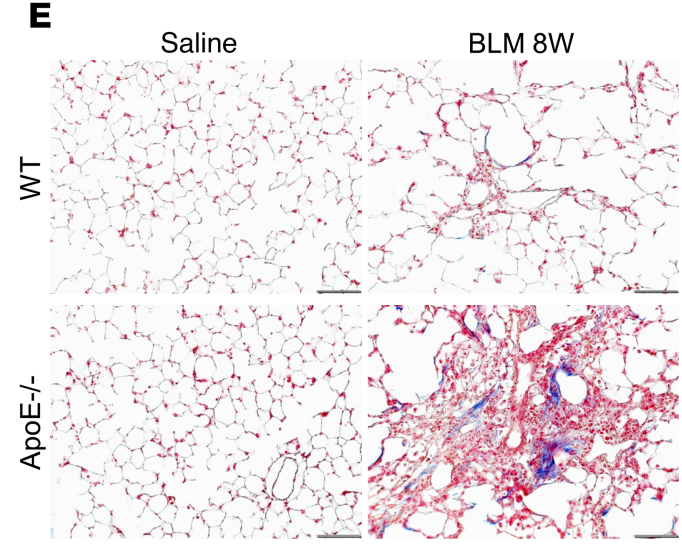
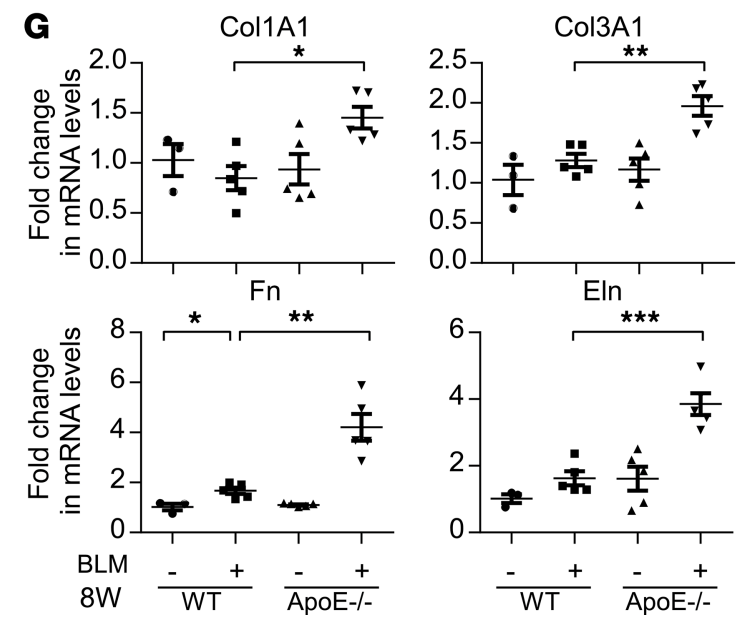

Figure 3. ApoE is required for the resolution of bleomycin-induced pulmonary fibrosis. (A) Six-week-old WT and ApoE ${ }^{-/-}$male mice were i.t. instilled with saline or bleomycin (BLM, $1.5 \mathrm{U} / \mathrm{kg}$ in $50 \mu \mathrm{L}$ saline). Eight weeks after treatment, mice were sacrificed for BALF harvesting, AM isolation, and fibrosis evaluation. (B and $\mathbf{C})$ ApoE levels in BALF (B) and AMs (C) were determined by ELISA and real-time PCR. $n=3$ mice for each group. mean \pm SEM; ${ }^{*} P<0.05$, ${ }^{*} P<0.01$ by 2-tailed Student's $t$ test. ( $\mathbf{D}$ and $\mathbf{E}$ ) Representative images of histology (D) and Masson's trichrome staining for collagens $(\mathbf{E})$ of the lungs from this experiment are shown. Original magnification, $\times 40$ (D), and $\times 200$ (E). Scale bars: $500 \mu \mathrm{m}$ (D), and $100 \mu \mathrm{m}$ (E). (F) The levels of hydroxyproline in the lungs were determined. $n=5,11,4,12$ mice for WT Saline, WT BLM, ApoE ${ }^{-/-}$Saline, and ApoE ${ }^{-l-} B L M$, respectively; mean $\pm S E M$; ${ }^{*} P<0.05$, ${ }^{* * *} P<$ 0.001 by 1 -way ANOVA with Bonferroni's post hoc test. (C) Total RNAs of the lungs were purified, and the expression of the indicated genes was assessed by real-time PCR. $n=3,5,5,5$ mice for WT Saline, WT BLM, ApoE ${ }^{-1-}$ Saline, and ApoE ${ }^{-1-}$ BLM, respectively; mean \pm SEM; ${ }^{*} P<0.05,{ }^{* *} P<0.01,{ }^{* * *} P<$ 0.001 by 1-way ANOVA with Bonferroni's post hoc test.

breakdown and clearance of excessive extracellular matrix components in the fibrotic lung, such as collagens, are believed to be among the necessary steps for this process (27). Therefore, we first determined whether ApoE affected lung Fb differentiation to myofibroblast. As shown in Figure 4, A and $\mathrm{B}$, neither purified ApoE protein nor conditioned media containing overexpressed ApoE had an effect on TGF- $\beta 1$-induced myofibroblast differentiation. Furthermore, ApoE did not show an effect on the dedifferentiation of myofibroblasts, either (Figure 4, C and D). Together, these data suggest that the proresolving activity of ApoE is unrelated to phenotypic switches of myofibroblasts in this phase. 
Given that macrophages are the primary phagocytes in mouse lungs (28), we then hypothesized that ApoE produced by Mo-AMs may play a role in the phagocytosis of collagens by these cells. We first observed that the expression of ApoE and type I Collagen largely converged and was colocalized in the fibrotic areas in the lungs of both bleomycin-treated mice and human IPF patients (Figure 5, A and B), suggesting a potential physical interaction. To test this hypothesis, we employed several independent methodologies. As shown in Figure 5C, type I Collagen in the conditioned media from myofibroblasts could bind to the immobilized recombinant ApoE in a dose-dependent manner. Additionally, immunoprecipitation of type I Collagen showed that it was associated with ApoE, also in a dose-dependent manner (Figure 5D). Via a solid-phase ELISA experiment, we found that ApoE bound to precoated type I Collagen, but not the control BSA (Figure 5E). Furthermore, the interaction of ApoE with the precoated type I Collagen could be competitively inhibited by free Collagen I (Figure 5F). Collectively, these experiments demonstrate an interaction between ApoE and type I Collagen.

ApoE promotes type I Collagen phagocytosis by macrophages, which is dependent on LRP1. Having shown the colocalization of ApoE and Collagen I in fibrotic lungs, and the direct binding of ApoE to type I Collagen, we then tested whether ApoE affected the phagocytosis of collagen by macrophages. We found via flow cytometry that macrophages could readily uptake FITC-labeled type I Collagen (Figure 6, A and B). However, this capability was significantly enhanced by exogenous ApoE (Figure 6, A and B). Such an augmentation by ApoE appears to be specific, as ApoE did not affect macrophage uptake of FITC-conjugated albumin (Figure 6B). To determine if ApoE affected the phagocytosis of collagens in vivo, we i.t. administered FITC-labeled type I Collagen into the lung of bleomycin-treated WT and $\mathrm{ApoE}^{-/-}$mice and found that engulfment of type I Collagen by AMs in the fibrotic lung was significantly diminished in the ApoE $\mathrm{E}^{-/-}$mice, as compared with that in WT animals (Figure 6, C and D). Despite ApoE promotion of collagen engulfment by macrophages, the altered phagocytosis demonstrated no effect on the expression of the profibrotic phenotype or mediators of these cells, either in vitro or in vivo (Supplemental Figure 2, A and B). Together, these data suggest that ApoE promotes the resolution of lung fibrosis by augmenting macrophage uptake of collagens.

The binding of ApoE to type I Collagen alone cannot fully account for how ApoE augments collagen engulfment by macrophages because ApoE itself is not a receptor but, rather, a secreted ligand. However, because ApoE mediates the clearance of LDL particles in circulation by vascular endothelial cells via ApoE interaction with its primary receptor, LRP1 (22), we speculated that LRP1 was required for the ApoE enhancement of collagen uptake. We first confirmed that ApoE did bind to the macrophage surface (Figure 6E). More importantly, this binding was inhibited by the natural LRP1 antagonist, LRPAP1 (29), or the synthetic peptide COG133, which is derived from the receptor-binding region of ApoE (residues 133-149) (30, 31), suggesting a reliance of the binding on this receptor (Figure 6E). However, neither LRPAP1 nor COG133 affected the binding of ApoE to Collagen I (Figure 6F). We next proceeded to examine the role of LRP1 in ApoE-associated collagen phagocytosis and found that blocking LRP1 by LRPAP1 or COG133 almost abolished the ApoE augmentation of type I Collagen engulfment (Figure 6, G-I). In summary, our findings suggest that the association of ApoE with collagen facilitates the uptake of the latter via the ApoE receptor LRP1.

Interference of the ApoE/LRP1 interaction impairs the resolution of pulmonary fibrosis. The demonstrations that the LRP1-binding ApoE mimetic COG133 hindered the ApoE binding to macrophage surface and impeded its promotion of collagen uptake by these cells indicate a possible negative impact of COG133 on the resolution of lung fibrosis in vivo. To test this hypothesis, we performed experiments as such: beginning at 4 weeks after i.t. bleomycin treatment, a time point at which established lung fibrosis starts to abate, mice were administered either COG133 or vehicle for an additional 4 weeks, followed by lung fibrosis evaluation (Figure 7A). We found that COG133 did blunt the resolution of lung fibrosis, as indicated by the generally increased hydroxyproline and profibrotic mediators in the lungs and BALF of bleomycin-treated mice that received this peptide (Figure 7, B-E). Collectively, these data suggest that ApoE/LRP1-mediated collagen clearance is critical to the resolution of this pathology.

Exogenous ApoE promotes the resolution of pulmonary fibrosis. We found that $\mathrm{ApoE}^{-/}$mice were deficient in resolving bleomycin-induced lung fibrosis. We also showed that Mo-AMs are the primary source of pulmonary ApoE in WT mice. These findings prompted us to ask if supplementing the lung with exogenous ApoE could improve the resolution of lung fibrosis in vivo. To test this hypothesis, $\mathrm{ApoE}^{-/-}$mice were injected with saline or bleomycin. Starting at 4 weeks after the treatment, mice were administered i.t. with exogenous ApoE protein (Figure 8A). 
A

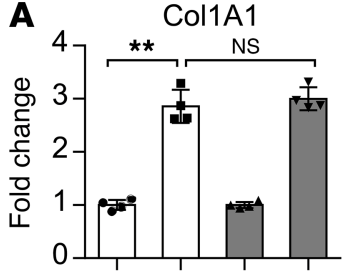

$\mathrm{Fn}$

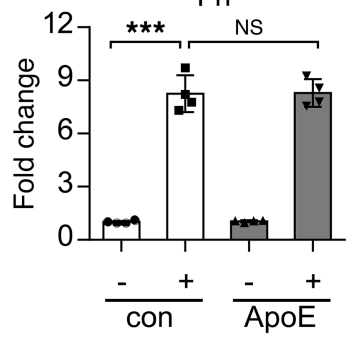

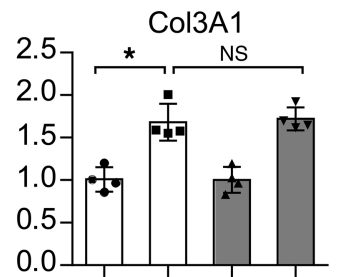

$\alpha$-SMA

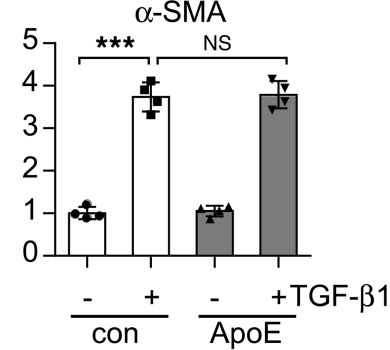

C

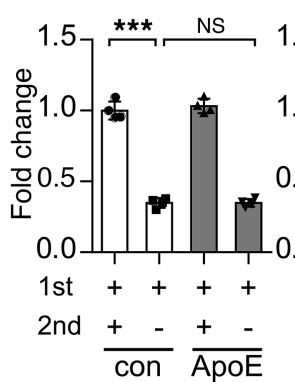

Fn

$\star \star \star * N S$

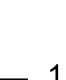

$\alpha$-SMA

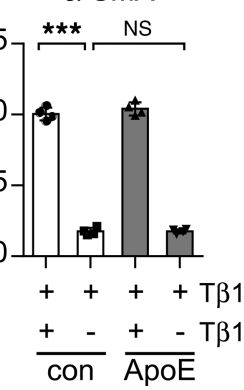

B

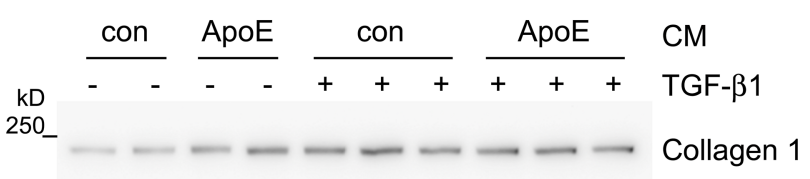

$250+-\cdots-\cdots+\cdots$

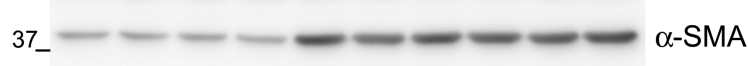

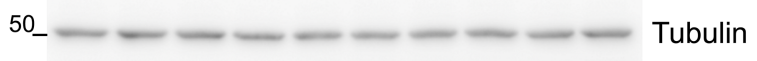

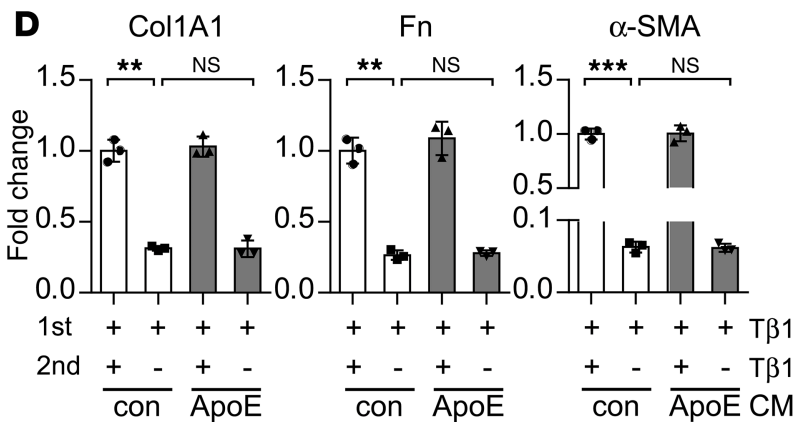

Figure 4. ApoE does not affect lung myofibroblast differentiation. (A) Human lung Fb MRC5 were treated with $2 \mathrm{ng} / \mathrm{mL}$ TGF- $\beta 1$ together with or without $1 \mu \mathrm{g} / \mathrm{mL}$ human ApoE for 48 hours. Total RNAs were isolated and levels of the indicated genes determined by real-time PCR. (B) MRC5 cells were treated with $2 \mathrm{ng} / \mathrm{mL}$ TCF- $\beta 1$ in conditioned media (CM) with or without ApoE. Forty-eight hours after treatment, cells were collected and protein levels of indicated genes determined by Western blotting. $n=3$ for each group. (C and D) MRC5 cells were treated with $2 \mathrm{ng} / \mathrm{mL}$ TCF- $\beta 1$ for 48 hours (1st T $\beta 1$ ) to induce myofibroblast differentiation. Myofibroblasts were washed 3 times and recultured in fresh control media or TCF- $\beta 1-$ containing media (2nd T $\beta 1$ ) with or without $1 \mu \mathrm{g} / \mathrm{mL} \mathrm{ApoE} \mathrm{(C),} \mathrm{or} \mathrm{conditioned} \mathrm{media} \mathrm{with} \mathrm{or} \mathrm{without} \mathrm{ApoE} \mathrm{(D)} \mathrm{for} \mathrm{additional} 48$ hours. Total RNAs were isolated and levels of the indicated genes determined by real-time PCR. $n=3-4$ for each group; mean \pm SD; ${ }^{*} P<0.05,{ }^{*} P<0.01,{ }^{* * *} P<0.001$ by 1-way ANOVA with Bonferroni's post hoc test.

As shown in Figure 8, B-E, ApoE supplementation promoted the resolution of lung fibrosis, as evidenced by the significantly reduced hydrxyproline and profibrotic mediators in the lungs and BALF of these mice. Given the implication of defective resolution of fibrosis in the pathogenesis of IPF, these data clearly suggest pulmonary ApoE supplementation as a therapeutic strategy for treating this pernicious disease.

\section{Discussion}

It has long been known that AMs in fibrotic lungs are derived from different origins and that these cells play an essential role in the disease pathogenesis (8). However, not until recently have cell lineage ablation studies compellingly shown that the ontogenetically distinct AMs demonstrate inequality in their contribution to the fibrotic response $(8,10,16-18)$. As an example, Mo-AMs, but not TR-AMs, are required for the development of maximal fibrosis in bleomycin-treated mouse lung (8). Nonetheless, the role of Mo-AMs in lung fibrosis resolution is still ambiguous. At least 2 studies showed that depletion of AMs actually impedes this homeostatic process $(19,20)$. Although both Mo-AMs and TR-AMs were indiscriminately depleted in those studies, the data indicate that Mo-AMs may have different or even opposite functions in the 2 phases of the bleomycin injury responses. Our finding that ApoE promotes lung fibrosis resolution has provided insight into this observation.

However, one may argue that a conditional ablation of ApoE in Mo-AMs, but not the global ApoE-KO used in this study, could provide more definitive evidence that it is Mo-AM-derived ApoE that promotes fibrosis resolution. Although we recognize the superiority of a conditional deletion in studying the function of a globally expressed gene, it has no significant advantage here because our data show that ApoE is almost exclusively produced by Mo-AMs in the injured lung. 
A
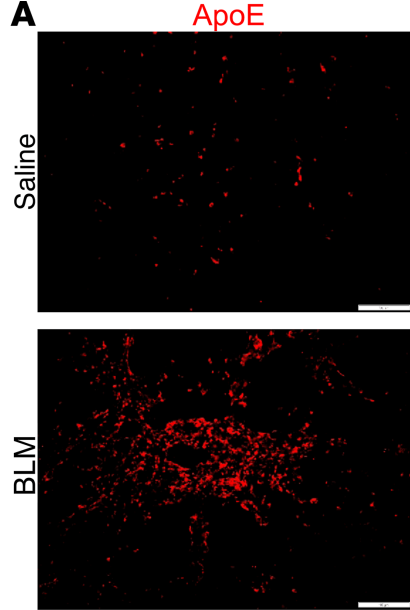

B
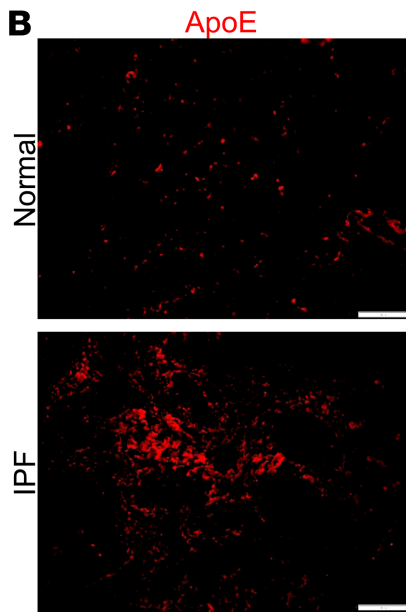

C
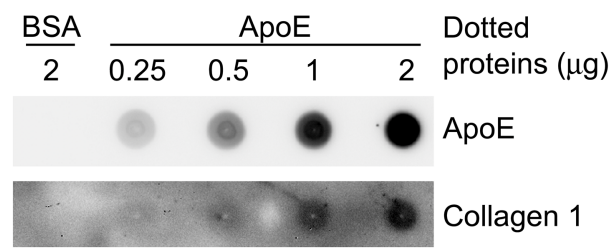

Collagen 1

E

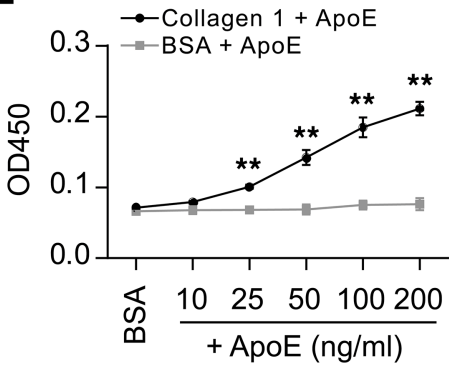

Collagen 1
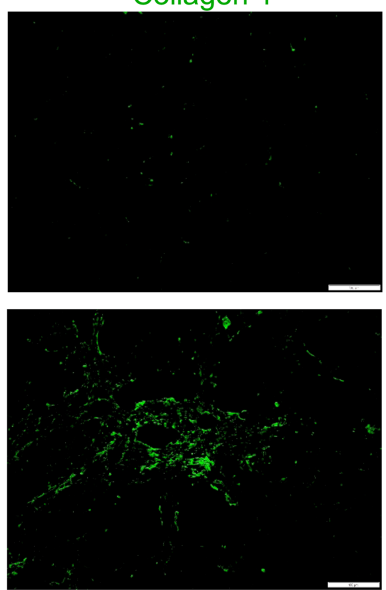

Collagen 1
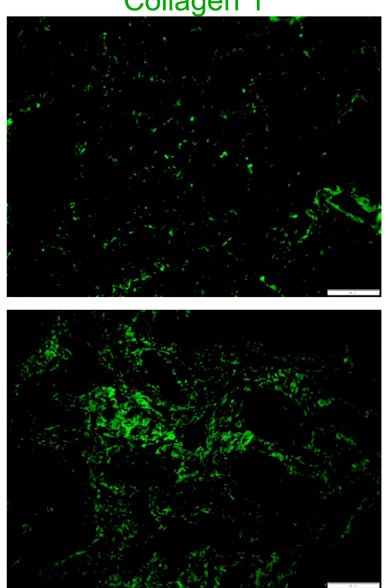

D

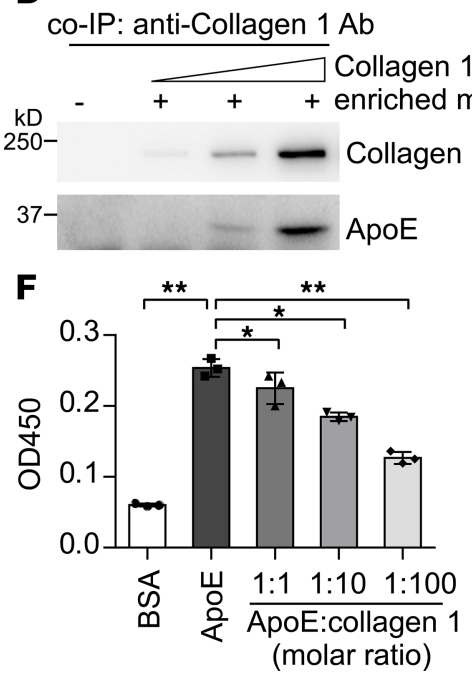

Overlay
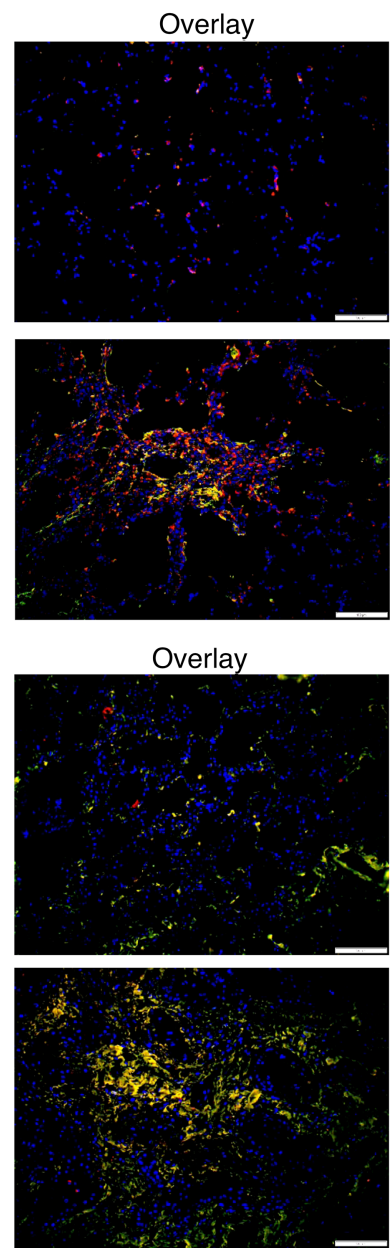

Figure 5. ApoE directly binds to type I

Collagen. (A) Eight-week-old C57BL/6 mice were i.t. instilled with saline or bleomycin (BLM, $1.5 \mathrm{U} / \mathrm{kg}$ in $50 \mu \mathrm{L}$ saline). Three weeks after treatment, mice were sacrificed and lung slices prepared. Immunofluorescence staining and fluorescence microscopy were performed to determine the expression of ApoE and Collagen I. Nuclei were counterstained with DAPI. Original magnification, $\times 200$. Scale bars: 100 $\mu \mathrm{m}$. (B) Slices of normal control and IPF lungs were prepared. Immunofluorescence staining and fluorescence microscopy were performed to determine the expression of ApoE and Collagen I. Nuclei were counterstained with DAPI. Original magnification, $\times 200$. Scale bars: 100 $\mu \mathrm{m}$. (C) Increasing amounts of recombinant human ApoE protein or BSA were dotted on a nitrocellulose membrane. After blocking, the membrane was incubated with the conditioned media from TGF- $\beta 1$-induced human lung myofibroblasts that are enriched with type I Collagen. Bound proteins on the membrane were determined by immunoblotting. (D) A constant amount of ApoE $(1 \mu \mathrm{g})$ was incubated with the increasing volumes of the conditioned media from TGF- $\beta 1$-induced lung myofibroblasts. Collagen I was then immunoprecipitated by anti-Collagen I antibody and ApoE in the immunocomplex was determined by Western blotting. (E) A 96-well high-binding plate was precoated with $10 \mu \mathrm{g} / \mathrm{mL}$ rat tail Collagen I or BSA overnight at $4^{\circ} \mathrm{C}$. Wells were washed and incubated with $200 \mathrm{ng} / \mathrm{mL}$ BSA or increasing amounts of purified human ApoE protein for 2 hours at room temperature, followed by subsequent incubation with primary ApoE antibody (1:2000 dilution) and HRP-conjugated secondary antibody (1:2000 dilution). Colorimetric development was achieved by TMB reaction. The plate was read at $450 \mathrm{~nm}$ in a 96-well plate reader. $n=3$; mean $\pm S D$; ${ }^{*} P<$ 0.01 compared with the BSA group. Two-tailed Student's $t$ test. (F) A 96-well high-binding plate was precoated with $10 \mu \mathrm{g} / \mathrm{mL}$ rat tail Collagen I overnight at $4^{\circ} \mathrm{C}$. Wells were washed and incubated with $50 \mathrm{ng} / \mathrm{mL}$ BSA or $50 \mathrm{ng} /$ $\mathrm{mL} A$ poE together with increasing amount of soluble Collagen I protein. Solid-binding assays were performed as in E. $n=3$; mean $\pm \mathrm{SD} ;{ }^{*} P<0.05,{ }^{*} P<0.01$ by 1 -way ANOVA with Bonferroni's post hoc test.

The primary function of ApoE is to promote the clearance of LDL and VLDL particles from the circulation and, thereby, mediate cholesterol metabolism (21). ApoE ${ }^{-/-}$mice develop hypercholesterolemia at adult age (21). This information may lead one to wonder if hypercholesterolemia contributes to the impaired resolution of lung fibrosis observed in these mice. Although we acknowledge that this hypothesis is justified, we believe that this scenario is less likely because (a) there has been no evidence supporting an association between the incidence of lung fibrosis and hypercholesterolemia in humans and (b) our study on PCSK9-KO mice, which have low blood cholesterol (32), indicates no positive correlation between the level of cholesterol and the severity of bleomycin-induced lung fibrosis (data not shown). 
A

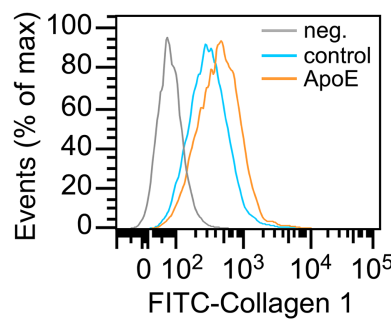

B
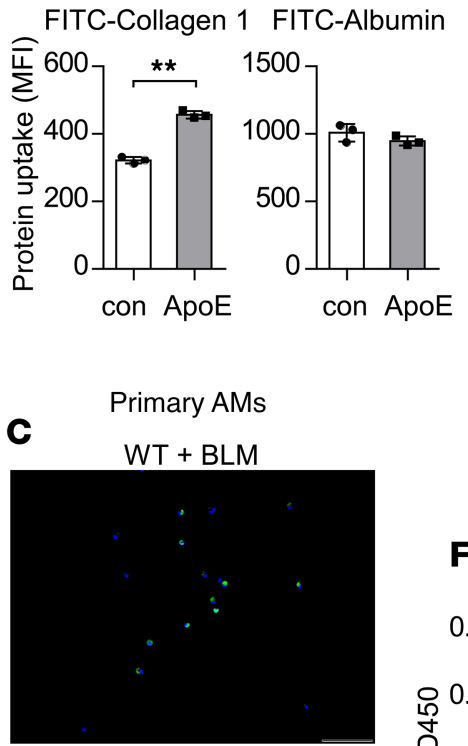

ApoE-/- + BLM

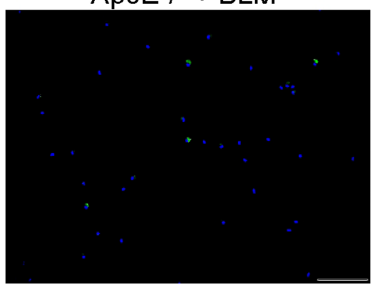

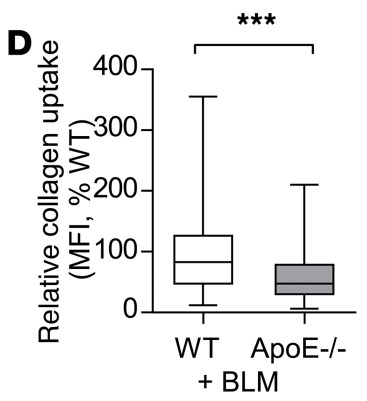

E
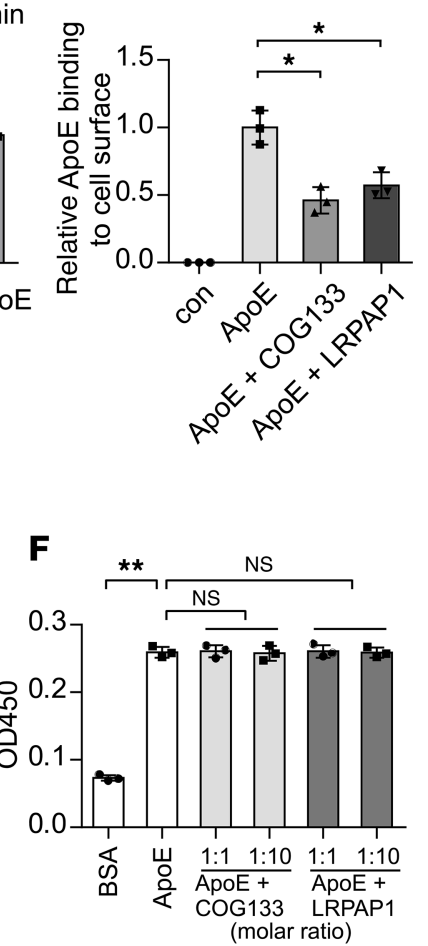

G

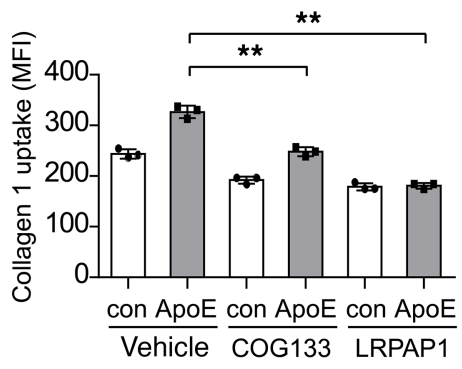

H

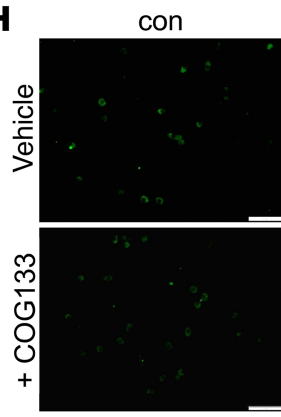

ApoE
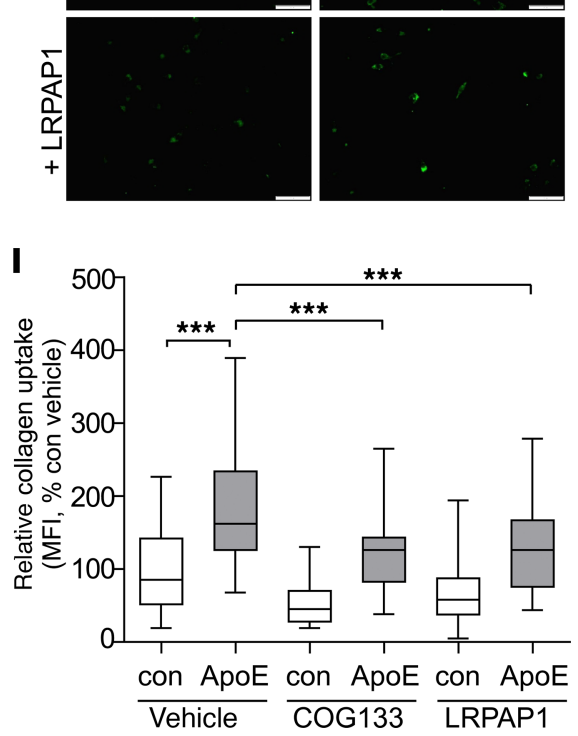

Figure 6. ApoE promotes type I Collagen phagocytosis by macrophages, which is dependent on LRP1. (A and B) FITC-conjugated type I Collagen or albumin $(50 \mu \mathrm{g} / \mathrm{mL})$ was preincubated with conditioned media with or without mouse ApoE. The mixtures were then incubated with mouse alveolar macrophages for 4 hours. After extensive wash, flow cytometry was performed in the presence of $0.02 \%$ trypan blue. Cells without incubation with Collagen I were used as a negative control (neg.) (A). Collagen I or albumin uptake was reflected by mean fluorescence index (MFI) $(\mathbf{B})$. $n=3$; mean $\pm \mathrm{SD}$; ${ }^{* *} P<0.01$ by 2-tailed Student's $t$ test. (C) Six-week-old WT and ApoE ${ }^{-1-}$ male mice were i.t. instilled with BLM. Four weeks later, mice were i.t. injected with $50 \mu \mathrm{g}$ FITC-conjugated type I Collagen. Two hours later, AMs were isolated, and representative images of the AMs demonstrating Collagen I uptake are shown. Original magnification, $\times 200$. Scale bars: $100 \mu \mathrm{m}$. (D) Quantitation of single cell fluorescence intensity was performed by ImageJ with a minimum of 200 cells analyzed and average calculated. Relative levels of Collagen I uptake are shown. ${ }^{* * *} P<0.001$ by 2 -tailed Student's $t$ test. (E) Alveolar macrophages were preincubated with $2 \mu \mathrm{g} / \mathrm{mL}$ BSA, COG133, or LRPAP1 for 30 minutes. The cells were then incubated with conditioned media with or without ApoE for 30 minutes. After extensive wash, cell surface-bound ApoE was determined by flow cytometry. $n=3$ for each group; mean \pm SD. (F) A 96-well high-binding plate was precoated with $10 \mu \mathrm{g} / \mathrm{mL}$ Collagen I overnight at $4^{\circ} \mathrm{C}$. Wells were washed and incubated with $50 \mathrm{ng} / \mathrm{mL}$ BSA or $50 \mathrm{ng} / \mathrm{mL}$ ApoE together with the indicated amounts of COG133 or LRPAP1, followed by incubation with ApoE antibody and HRP-conjugated secondary antibody and development with TMB substrate. $n=3$; mean \pm SD. (C) Alveolar macrophages were preincubated with $2 \mu \mathrm{g} / \mathrm{mL}$ BSA, COG133, or LRPAP1 for 30 minutes. The cells were then incubated with mixture of type I Collagen with control or ApoE conditioned media for 4 hours, followed by flow cytometry as in $\mathbf{A}$ and $\mathbf{B} . n=3$; mean \pm SD. (H and I) Alveolar macrophages were treated as in G. Original magnification, $\times 400$. Scale bars: $50 \mu \mathrm{m}(\mathbf{H})$. Quantitation of single cell fluorescence intensity was performed by Image and average calculated. Relative levels of Collagen I uptake are shown (I). ${ }^{*} P<0.05,{ }^{* *} P<0.01,{ }^{* *} P<0.001$ by 1 -way ANOVA with Bonferroni's post hoc test (E-I). The box-and-whisker plots depict the 25 th and 75 th percentiles and median, minimum, and maximum values (D and I). 
A

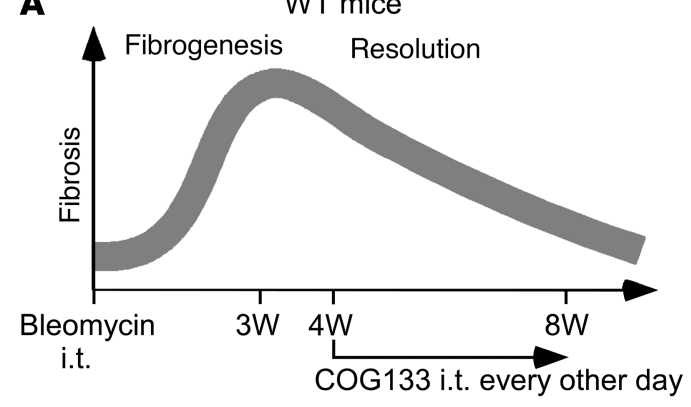

B Mouse lungs

hydroxyproline content

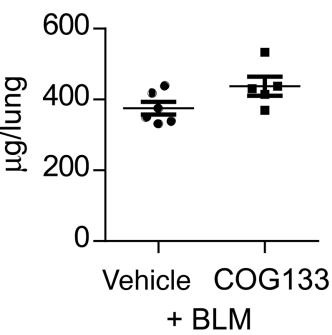

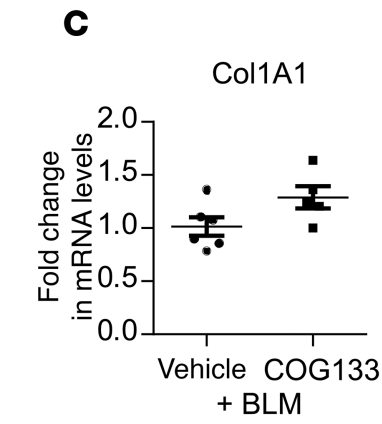

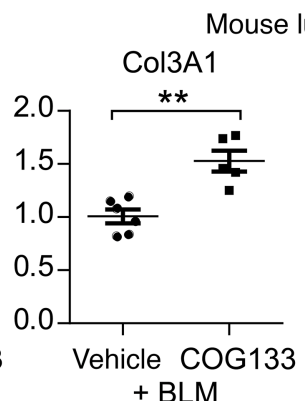

Mouse lungs

D

BALF from BLM treated mice
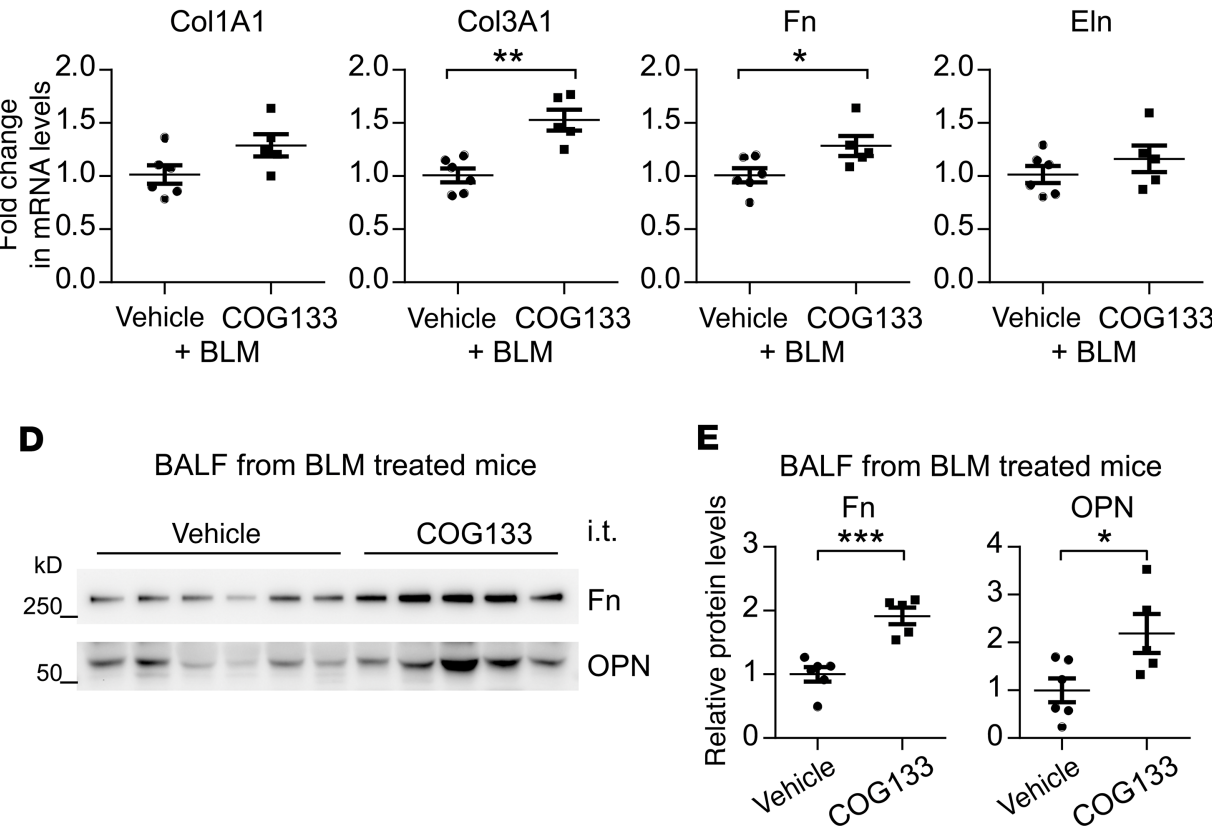

Figure 7. Interference of ApoE/LRP1 interaction impairs the resolution of pulmonary fibrosis. (A) Six-week-old WT male mice were i.t. instilled with bleomycin (BLM, $1.5 \mathrm{U} / \mathrm{kg}$ in $50 \mu \mathrm{L}$ saline). Starting at 4 weeks after bleomycin treatment, mice were administered i.t. COC133 (1 mg/kg in $50 \mu \mathrm{L}$ saline) or saline alone, once every other day. Mice were sacrificed at 8 weeks after bleomycin injection for fibrosis evaluation. (B) The levels of hydroxyproline in the lungs were determined. (C) Total RNAs of the lungs were purified, and the expression of the indicated genes was assessed by real-time PCR. (D and E) BALF protein was precipitated and resolved by SDS-PAGE. Levels of the indicated proteins in BALF were determined by Western blotting (D) and densitometric analyses performed using ImageJ (E). $n=6,5$; mean \pm SEM; ${ }^{*} P<0.05,{ }^{* *} P<0.01,{ }^{* *} P<0.001$ by 2 -tailed Student's $t$ test (B-E).

Given that ApoE promotes collagen uptake, one may wonder why $\mathrm{ApoE}^{-/-}$mice do not develop more severe lung fibrosis than WT animals at 3 weeks after bleomycin injury. We believe that this might be attributed to two scenarios: (a) Although ApoE is mainly produced by Mo-AMs, the profibrotic activity of these cells simply overpowers any of their beneficial effects during the time period, leading to a full-blown fibrosis in the lung, and (b) ApoE promotion of collagen engulfment is dependent on the receptor LRP1, whereas the LRP1 level and/or activity at the early fibrogenetic phase may not be conducive to this process. These possibilities are also reflecting the reported difference between the roles of Mo-AMs at the fibrosis developing and resolving stages. Regardless, although ApoE seems not to have an effect on progressing to the maximal fibrosis in the lung, it is still worth examining what the function of this Mo-AM-produced molecule is during this time period simply because of its large quantity.

Additionally, one inference from LRP1 mediation of ApoE-dependent collagen phagocytosis is that pulmonary LRP1 in $\mathrm{ApoE}^{-/-}$mice in the fibrotic resolving phase is greater than that in WT animals, while ApoE supplementation will lead to its decline. This reasoning appears incorrect because the levels of pulmonary LRP1 were found unchanged in those settings (Supplemental Figure 3, A-D). 
A

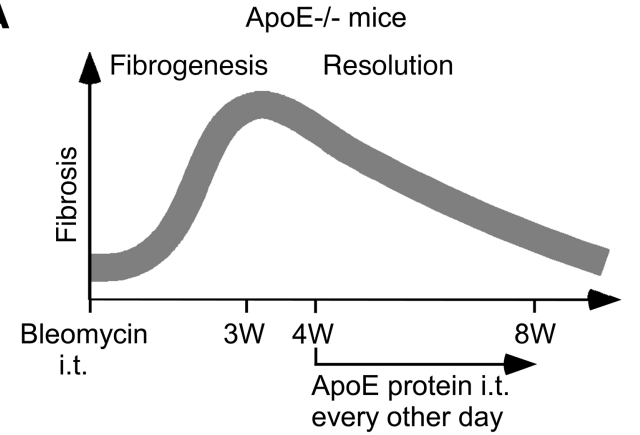

B ApoE-/- mouse lungs hydroxyproline content

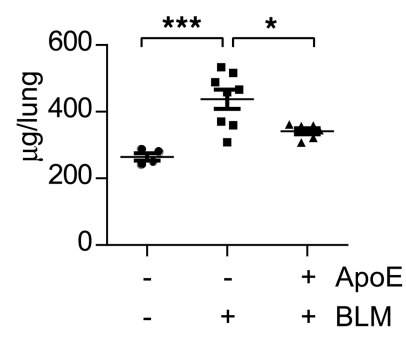

C

ApoE-/- mouse lungs
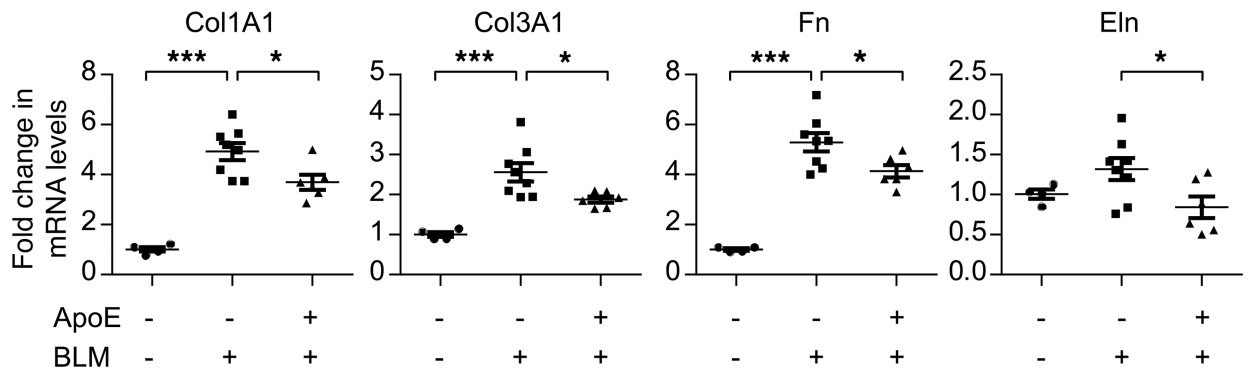

D

BALF from BLM treated ApoE-/- mice

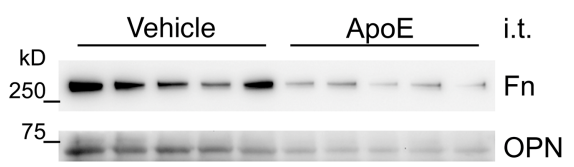

E BALF from BLM treated ApoE-/- mice

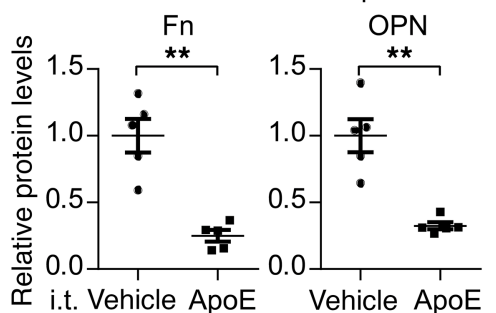

Figure 8. Exogenous ApoE promotes the resolution of pulmonary fibrosis. (A) Six-week-old ApoE $\mathrm{E}^{-/-}$male mice were i.t. instilled with saline or bleomycin (BLM, $1.5 \mathrm{U} / \mathrm{kg}$ in $50 \mu \mathrm{L}$ saline). Starting at 4 weeks after bleomycin treatment, mice were treated i.t. ApoE ( $1 \mu \mathrm{g}$ in $50 \mu \mathrm{L}$ saline) or saline alone, once every other day. Mice were eventually sacrificed at 8 weeks after bleomycin injection for lung harvesting. (B) The levels of hydroxyproline in the lungs were determined. $n=4,8,6$ mice for $\mathrm{BLM}^{-} \mathrm{ApoE} \mathrm{E}^{-}, \mathrm{BLM}^{+} \mathrm{ApoE}-$, and $\mathrm{BLM}^{+} A \mathrm{PoE}^{+}$, respectively; mean $\pm \mathrm{SEM} ;{ }^{*} P<0.05,{ }^{* *} P<0.001$ by 1-way ANOVA with Bonferroni's post hoc test. (C) Total RNA of the lungs was purified and the expression of the indicated genes was assessed by real-time PCR. $n=4,8,6$ mice for $\mathrm{BLM}^{-} \mathrm{ApoE}^{-}, \mathrm{BLM}^{+} \mathrm{ApoE} \mathrm{E}^{-}$, and $\mathrm{BLM}^{+} \mathrm{ApoE}^{+}$, respectively; mean $\pm \mathrm{SEM}$; ${ }^{*} P<0.05,{ }^{* * *} P<0.001$ by 1 -way ANOVA with Bonferroni's post hoc test. ( $\mathbf{D}$ and $\mathbf{E}$ ) BALF protein was precipitated and resolved by SDS-PAGE. Levels of the indicated proteins in BALF were determined by Western blotting (D) and densitometric analyses performed using Imagej (E). $n=5,5$ for Vehicle and ApoE, respectively; mean $\pm \mathrm{SEM} ;{ }^{* *} P<0.01$ by 2 -tailed Student's $t$ test.

However, such an observation is apparently consistent with past findings that LRP1 can be continuously endocytosed from the membrane and recycled back to the cell surface (33).

Our data show that pulmonary ApoE is upregulated in IPF patients, as compared with those in control subjects. Although consistent with the facts that ApoE is almost exclusively produced by Mo-AMs and that there are few Mo-AMs in healthy lungs, this finding appears counterintuitive because one would ask why lung fibrosis still occurs despite the elevation of pulmonary ApoE. This is likely due to the fact that Mo-AM production of ApoE is a negative-feedback mechanism by which all hosts are equipped in response to fibrotic injuries to the lung, whereas only those who come up with insufficient or defective ApoE are to develop lung fibrosis. Therefore, future studies should focus on identifying a potential correlation between pulmonary ApoE levels and the clinicopathological parameters with IPF patients.

It has been also well documented that ApoE polymorphism is closely associated with some neurodegenerative disorders (34-36). In fact, the E4 variant of ApoE is the widest known genetic risk factor for late-onset sporadic Alzheimer's disease (AD) (34-37). Although the underlying mechanism by which these disease-associated ApoE variants participate in the pathogenesis remains incompletely understood, there 
have been already-ample evidence showing that the pathogenic ApoE leads to an abnormal response of microglia to amyloid deposition in the brain $(34,36,38)$. Currently, there is no evidence indicating a causal link between ApoE polymorphism and lung fibrosis (39). However, given that organ fibrosis, including IPF, normally occurs in the aged population, much like most of the neurodegenerative diseases, we are certainly intrigued to undertake such an investigation in future studies.

Finally, it should be noted that there is an elevated fibrotic pathology in $\mathrm{ApoE}^{-/-}$mice even at 8 week after bleomycin injury; the relevant cells that reside in that profibrotic environment, such as myofibroblasts, may respond to such a setting by demonstrating a more pronounced profibrotic phenotype (upregulation of ECM production). With that being said, we have to acknowledge that we can't absolutely rule out potential mechanisms other than the regulation of fibrosis resolution.

In summary, our study has provided evidence that Mo-AM-derived ApoE is beneficial to the resolution of lung fibrosis, therefore establishing a strong rationale for targeting ApoE to treat this disease to which efficacious remedy remains scarce.

\section{Methods}

Reagents. Human plasma-derived ApoE and recombinant ApoE3 proteins were purchased from BioVision. ApoE mimetic peptide COG133 was from APExBIO. Recombinant mouse LRPAP1 protein was from R\&D Systems. FITC-conjugated type I Collagen from bovine skin and FITC-conjugated albumin were from Sigma-Aldrich. Rat tail Collagen I protein was from Thermo Fisher Scientific. Bleomycin was from Hospira.

Experimental pulmonary fibrosis model. Six- to 8-week-old male C57BL/6 mice and ApoE-KO mice (B6.129P2-Apoe $\mathrm{tm}^{\mathrm{m} 1 \mathrm{Un}} / \mathrm{J}$ ) were purchased from The Jackson Laboratory. The i.t. bleomycin lung fibrosis mouse model has been previously detailed (40).

Cell lines. Human lung Fb line MRC-5 and mouse AM line MH-S were purchased from American Type Culture Collection.

Human lung tissues. IPF and failed donor normal lung tissues were obtained from the University of Alabama Birmingham Tissue Procurement and Cell Culture Core.

Isolation of primary mouse AMs, AECs, and lung Fbs. Mouse AMs were purified as previously described (41). Briefly, cells within BALF were collected by centrifugation (500 $g$ for 5 minutes). After RBC lysis, BAL cells were plated for 1 hour, followed by extensive wash to remove unattached cells. The attached cells were used as AMs. To isolate Mo-AMs and TR-AMs, BAL cells were stained with a cocktail of antibodies including PerCP/Cy5.5 anti-CD64 (BioLegend, clone X54-5/7.1), eFluor 450 anti-F4/80 (clone BM8), Alexa Fluor 488 anti-CD11b (clone M1/70) and eFluor 660 anti-CD170 (Siglec F) (clone 1RNM44N), all from eBioscience. Resident $\left(\mathrm{CD} 64^{+} \mathrm{F} 4 / 80^{+}\right.$Siglec $\left.\mathrm{F}^{\mathrm{hi}} \mathrm{CD} 11 \mathrm{~b}^{\mathrm{lo}}\right)$ and Mo-AMs $\left(\mathrm{CD} 64^{+} \mathrm{F} 4 / 80^{+}\right.$Siglec $\mathrm{F}^{\mathrm{lo}} \mathrm{CD}$ $11 \mathrm{~b}^{\mathrm{hi}}$ ) were isolated by fluorescence activated cell sorting.

Primary mouse AECs and lung Fbs were isolated, as previously described, with minor modifications (42). Briefly, lungs were minced and digested in HBSS containing $0.1 \%$ type I collagenase (Worthington), $0.1 \%$ dispase II (Sigma-Aldrich), and $0.01 \%$ DNase I (Worthington). Lung digests were passed through a $40-\mu \mathrm{m}$ mesh cell strainer, RBC were lysed, and residual cells were pelleted. After resuspension, cells were incubated with a mixture of biotin-conjugated anti-CD16/32 (clone 2.4G2), -CD45 (clone 30-F11), and -CD31 (clone MEC 13.3) antibodies (all from BD Biosciences) and streptavidin-conjugated magnetic beads (Promega) to deplete myeloid and endothelial cells. AECs and lung Fbs were then isolated from the cell suspensions with streptavidin-conjugated magnetic beads coupled with biotin-conjugated anti-EpCAM (clone G8.8) and anti-PDGFR- $\alpha$ (clone APA5) antibodies (both from BD Biosciences), respectively.

Hydroxyproline determinations. The right 3 (superior, middle, and inferior) lobes and the left mouse lung were homogenized in $2 \mathrm{~mL} \mathrm{H}_{2} \mathrm{O}$. A total of $100 \mu \mathrm{L}$ homogenates were mixed with $100 \mu \mathrm{L} 12 \mathrm{~N} \mathrm{HCl}$, and the samples incubated at $120^{\circ} \mathrm{C}$ for 3 hours. Hydroxyproline levels were then determined with BioVision's Hydroxyproline Assay Kit according to the manufacturer's instructions.

Histology and immunofluorescence staining. H\&E staining and Masson's trichrome staining for collagen depositions were performed as described previously (43). For immunofluorescence staining, paraffin sections of human or mouse lungs were deparaffinized and rehydrated, and antigen unmasked by boiling in citric acid-based antigen unmasking solution (Vector Laboratories, H-3300) for 15 minutes. Tissue sections were blocked in TBS containing 3\% BSA and 0.1\% Triton X-100, flowed by blocking with Fc Receptor Blocker reagent (Innovex). Sections were then stained with primary antibodies (1:100-1:500 dilution) and 
fluorochrome-conjugated secondary antibodies (1:500 dilution, Thermo Fisher Scientific). After wash, slides were treated with Vector TrueVIEW Autofluorescence Quenching kit (Vector Laboratories) and mounted with VECTASHIELD medium with DAPI (Vector Laboratories) for microscope imaging. Rabbit anti-ApoE antibodies were from Invitrogen (catalog 701241) and Abcam (catalog ab20874). Goat antiCollagen I antibody was from Southern Biotech (catalog 1310-01).

Real-time PCR. mRNA levels were determined by real-time PCR using SsoAdvanced Universal SYBR Green Supermix (Bio-Rad). Primer sequences are listed in Supplemental Table 1. To calculate fold change in the expression of these genes, $\Delta \mathrm{Ct}=\mathrm{Ct}$ of tubulin $-\mathrm{Ct}$ of individual genes was first obtained. $\Delta \Delta \mathrm{Ct}=\Delta \mathrm{Ct}$ of treated groups $-\Delta \mathrm{Ct}$ of untreated control groups was then obtained. Fold change was calculated as $2^{\Delta \Delta \mathrm{Ct}}$, with control groups as 1 fold.

Western blotting. Western blotting was performed as previously described (44). Mouse anti- $\alpha$-tubulin (catalog T5168) and anti- $\alpha$-SMA (catalog A5228) antibodies were from Sigma-Aldrich. Goat anti-Collagen I (catalog 1310-01) and rabbit anti-Collagen I (catalog ab138492) antibodies were from SouthernBiotech and Abcam, respectively. Rabbit anti-Fn antibody (catalog 15613-1-AP) was from Proteintech. Rabbit antiApoE antibodies were from Invitrogen (catalog 701241) and Abcam (catalog ab20874), and mouse antiOPN antibody (catalog sc-21742) was from Santa Cruz Biotechnology Inc. Densitometric analyses of blots were performed using ImageJ software (NIH). See complete unedited blots in the supplemental material.

ELISA. Levels of ApoE in mouse BALF or lung tissue extracts were determined using Mouse ApoE ELISA Kit (Abcam, catalog ab215086) according to the manufacturer's instructions.

Solid-phase binding assay. Ninety-six-well EIA/RIA high binding plates (Corning) were coated with $10 \mu \mathrm{g} / \mathrm{mL}$ rat tail Collagen I (Thermo Fisher Scientific) diluted in $0.01 \mathrm{~N}$ acetic acid overnight at $4^{\circ} \mathrm{C}$, followed by wash with PBS- $0.05 \%$ Tween 20 and blocking with HBSS-1\% BSA. Purified human ApoE protein diluted in HBSS-1\%BSA was added to wells for 2 hours at room temperature. Wells were washed and subsequently incubated with primary ApoE antibody (1:2000 dilution) and HRP-conjugated secondary antibody (1:2000 dilution). Colorimetric detection was achieved using TMB Substrate Kit (Thermo Fisher Scientific). The reaction was stopped with $2 \mathrm{~N} \mathrm{H}_{2} \mathrm{SO}_{4}$, and absorbance was measured at $450 \mathrm{~nm}$ in a 96-well plate reader (BioTek). For competitive binding assay, purified ApoE protein was incubated with an increasing amount of soluble Collagen I protein in Collagen I precoated plates, and assays were performed as described above.

CoIP. For CoIP of Collagen I and ApoE, goat anti-Collagen I antibody was bound to Dynabeads Protein G (Invitrogen). Beads were then incubated with purified human ApoE protein and Collagen Ienriched media from TGF- $\beta 1$-treated human lung Fbs. After incubation, beads were washed 3 times with RIPA buffer, and proteins were eluted from beads by $1 \%$ SDS lysis buffer, followed by separation by SDSPAGE and immunoblotting using anti-ApoE antibody.

Collagen uptake assay. Mouse AMs were incubated with $50 \mu \mathrm{g} / \mathrm{mL}$ FITC-conjugated Collagen I in serum free media for 4-6 hours. Cells were washed rigorously with PBS, trypsinized, and resuspended in PBS containing $1 \%$ BSA. Before analysis, cells were incubated with $0.02 \%$ trypan blue for $2-5$ minutes to quench the fluorescence of membrane-bound Collagen I, and internalized Collagen I levels were determined by flow cytometry. Alternatively, after FITC-conjugated Collagen I incubation, cells were washed, counterstained with DAPI, and assessed with fluorescence microscopy. For the in vivo uptake assay, mice were instilled i.t. with $50 \mu \mathrm{L}$ saline or FITC-conjugated Collagen I $(1 \mathrm{mg} / \mathrm{mL})$. BAL cells were collected 2 hours later, and the AMs were obtained after adhesion to tissue culture plates. Cells were then counterstained with DAPI and examined by fluorescence microscopy.

Statistics. One-way ANOVA followed by the Bonferroni test was used for multiple group comparisons. The 2-tailed Student's $t$ test was used for comparison between 2 groups. $P<0.05$ was considered statistically significant.

Study approval. All animal protocols were approved by the University of Alabama at Birmingham IACUC. The human protocol was approved by the University of Alabama at Birmingham IRB.

\section{Author contributions}

HC and DJ contributed equally to this work; HC and GL designed the study; HC, DJ, SB, NX, SRD, TK, RML, and GL performed the experiments and/or analyzed the data; and HC, SRD, and GL wrote the manuscript. 


\section{Acknowledgments}

This work was supported by NIH grant HL135830.

Address correspondence to: Gang Liu, Professor of Medicine, Division of Pulmonary, Allergy, and Critical Care Medicine, Department of Medicine, University of Alabama at Birmingham, 901 19th Street South, BMR II 233, Birmingham, Alabama 35294, USA. Phone: 205.975.8932; Email: gangliu@uabmc.edu.

1. Noble PW, Barkauskas CE, Jiang D. Pulmonary fibrosis: patterns and perpetrators. J Clin Invest. 2012;122(8):2756-2762.

2. Mora AL, Rojas M, Pardo A, Selman M. Emerging therapies for idiopathic pulmonary fibrosis, a progressive age-related disease. Nat Rev Drug Discov. 2017;16(11).

3. Glasser SW, Hagood JS, Wong S, Taype CA, Madala SK, Hardie WD. Mechanisms of Lung Fibrosis Resolution. Am J Pathol. 2016;186(5):1066-1077.

4. Philip K, et al. HIF1A up-regulates the ADORA2B receptor on alternatively activated macrophages and contributes to pulmonary fibrosis. FASEB J. 2017;31(11):4745-4758

5. Tao B, et al. Myeloid-specific disruption of tyrosine phosphatase Shp2 promotes alternative activation of macrophages and predisposes mice to pulmonary fibrosis. J Immunol. 2014;193(6):2801-2811.

6. Watanabe S, Alexander M, Misharin AV, Budinger GRS. The role of macrophages in the resolution of inflammation. J Clin Invest. 2019;129(7):2619-2628.

7. Wynn TA, Vannella KM. Macrophages in Tissue Repair, Regeneration, and Fibrosis. Immunity. 2016;44(3):450-462.

8. Joshi N, Walter JM, Misharin AV. Alveolar Macrophages. Cell Immunol. 2018;330:86-90.

9. Reyfman PA, et al. Single-Cell Transcriptomic Analysis of Human Lung Provides Insights into the Pathobiology of Pulmonary Fibrosis. Am J Respir Crit Care Med. 2019;199(12):1517-1536.

10. Aran D, et al. Reference-based analysis of lung single-cell sequencing reveals a transitional profibrotic macrophage. Nat Immunol. 2019;20(2):163-172.

11. Morse C, et al. Proliferating SPP1/MERTK-expressing macrophages in idiopathic pulmonary fibrosis. Eur Respir J. 2019;54(2):1802441

12. Misharin AV, Morales-Nebreda L, Mutlu GM, Budinger GR, Perlman H. Flow cytometric analysis of macrophages and dendritic cell subsets in the mouse lung. Am J Respir Cell Mol Biol. 2013;49(4):503-510.

13. Mould KJ, et al. Cell Origin Dictates Programming of Resident versus Recruited Macrophages during Acute Lung Injury. Am J Respir Cell Mol Biol. 2017;57(3):294-306.

14. Misharin AV, et al. Monocyte-derived alveolar macrophages drive lung fibrosis and persist in the lung over the life span. $J$ Exp Med. 2017;214(8):2387-2404.

15. Joshi N, et al. A spatially restricted fibrotic niche in pulmonary fibrosis is sustained by M-CSF/M-CSFR signalling in monocyte-derived alveolar macrophages. Eur Respir J. 2020;55(1):1900646.

16. McCubbrey AL, et al. Deletion of c-FLIP from CD11b ${ }^{\text {hi }}$ Macrophages Prevents Development of Bleomycin-induced Lung Fibrosis. Am J Respir Cell Mol Biol. 2018;58(1):66-78.

17. Meziani L, et al. CSF1R inhibition prevents radiation pulmonary fibrosis by depletion of interstitial macrophages. Eur Respir J. 2018;51(3):1702120

18. Moore BB, et al. Protection from pulmonary fibrosis in the absence of CCR2 signaling. J Immunol. 2001;167(8):4368-4377.

19. Cabrera S, et al. Delayed resolution of bleomycin-induced pulmonary fibrosis in absence of MMP13 (collagenase 3). Am J Physiol Lung Cell Mol Physiol. 2019;316(5):L961-L976.

20. Gibbons MA, et al. Ly6Chi monocytes direct alternatively activated profibrotic macrophage regulation of lung fibrosis. $A m J$ Respir Crit Care Med. 2011;184(5):569-581.

21. Silvestre-Roig C, de Winther MP, Weber C, Daemen MJ, Lutgens E, Soehnlein O. Atherosclerotic plaque destabilization: mechanisms, models, and therapeutic strategies. Circ Res. 2014;114(1):214-226.

22. Tachibana M, et al. APOE4-mediated amyloid- $\beta$ pathology depends on its neuronal receptor LRP1. J Clin Invest. 2019;129(3):1272-1277.

23. Xie N, et al. Metabolic characterization and RNA profiling reveal glycolytic dependence of profibrotic phenotype of alveolar macrophages in lung fibrosis. Am J Physiol Lung Cell Mol Physiol. 2017;313(5):L834-L844.

24. Ucero AC, et al. Fra-2-expressing macrophages promote lung fibrosis in mice. J Clin Invest. 2019;129(8):3293-3309.

25. Kim MS, et al. IL-37 Attenuates Lung Fibrosis by Inducing Autophagy and Regulating TGF- $\beta 1$ Production in Mice. J Immunol. 2019;203(8):2265-2275.

26. Hecker L, et al. Reversal of persistent fibrosis in aging by targeting Nox4-Nrf2 redox imbalance. Sci Transl Med. 2014;6(231):231ra47.

27. Atabai K, et al. Mfge8 diminishes the severity of tissue fibrosis in mice by binding and targeting collagen for uptake by macrophages. J Clin Invest. 2009;119(12):3713-3722.

28. Kim KK, et al. Efferocytosis of apoptotic alveolar epithelial cells is sufficient to initiate lung fibrosis. Cell Death Dis. 2018;9(11):1056.

29. Willnow TE, Sheng Z, Ishibashi S, Herz J. Inhibition of hepatic chylomicron remnant uptake by gene transfer of a receptor antagonist. Science. 1994;264(5164):1471-1474.

30. Lynch JR, et al. APOE genotype and an ApoE-mimetic peptide modify the systemic and central nervous system inflammatory response. J Biol Chem. 2003;278(49):48529-48533.

31. Azevedo OG, et al. Apolipoprotein E COG 133 mimetic peptide improves 5-fluorouracil-induced intestinal mucositis. BMC Gastroenterol. 2012;12:35. 
32. Nishikido T, Ray KK. Targeting the peptidase PCSK9 to reduce cardiovascular risk: Implications for basic science and upcoming challenges. Br J Pharmacol. 2019; https://doi.org/10.1111/bph.14851.

33. Lillis AP, Mikhailenko I, Strickland DK. Beyond endocytosis: LRP function in cell migration, proliferation and vascular permeability. J Thromb Haemost. 2005;3(8):1884-1893.

34. Huang YA, Zhou B, Wernig M, Südhof TC. ApoE2, ApoE3, and ApoE4 Differentially Stimulate APP Transcription and A $\beta$ Secretion. Cell. 2017;168(3):427-441.e21.

35. Cramer PE, et al. ApoE-directed therapeutics rapidly clear $\beta$-amyloid and reverse deficits in AD mouse models. Science. 2012;335(6075):1503-1506.

36. Yin C, et al. ApoE attenuates unresolvable inflammation by complex formation with activated C1q. Nat Med. 2019;25(3):496-506.

37. Shi Y, et al. ApoE4 markedly exacerbates tau-mediated neurodegeneration in a mouse model of tauopathy. Nature. 2017;549(7673):523-527.

38. Deane R, et al. apoE isoform-specific disruption of amyloid beta peptide clearance from mouse brain. J Clin Invest. 2008;118(12):4002-4013.

39. Yao X, Gordon EM, Figueroa DM, Barochia AV, Levine SJ. Emerging Roles of Apolipoprotein E and Apolipoprotein A-I in the Pathogenesis and Treatment of Lung Disease. Am J Respir Cell Mol Biol. 2016;55(2):159-169.

40. Liu G, et al. miR-21 mediates fibrogenic activation of pulmonary fibroblasts and lung fibrosis. J Exp Med. 2010;207(8):1589-1597.

41. Cui H, et al. Long noncoding RNA Malat1 regulates differential activation of macrophages and response to lung injury. JCI Insight. 2019;4(4):124522.

42. Cui H, et al. miR-34a promotes fibrosis in aged lungs by inducing alveolarepithelial dysfunctions. Am J Physiol Lung Cell Mol Physiol. 2017;312(3):L415-L424.

43. Yang S, et al. Participation of miR-200 in pulmonary fibrosis. Am J Pathol. 2012;180(2):484-493.

44. Song W, et al. Inhibition of lung fluid clearance and epithelial $\mathrm{Na}+$ channels by chlorine, hypochlorous acid, and chloramines J Biol Chem. 2010;285(13):9716-9728. 